\title{
Real-time merging traffic control for throughput maximization at motorway work zones
}

\section{Journal Article}

Author(s):

Tympakianaki, Athina; Spiliopoulou, Anastasia D.; Kouvelas, Anastasios (D); Papageorgiou, Markos

Publication date:

2012

Permanent link:

https://doi.org/10.3929/ethz-b-000275924

Rights / license:

Creative Commons Attribution-NonCommercial-NoDerivs 3.0 Unported

Originally published in:

Procedia-Social and Behavioral Sciences 48, https://doi.org/10.1016/j.sbspro.2012.06.1130 


\title{
Real-time merging traffic control for throughput maximization at motorway work zones
}

\author{
Athina Tympakianaki ${ }^{\mathrm{a},{ }^{*},}$, Anastasia D. Spiliopoulou ${ }^{\mathrm{a}}$, Anastasios Kouvelas ${ }^{\mathrm{a}}$, \\ Markos Papageorgiou ${ }^{\mathrm{a}}$
}

${ }^{a}$ Dynamic Systems and Simulation Laboratory, Technical University of Crete, 73100 Chania, Greece

\begin{abstract}
Work zones on motorways necessitate the drop of one or more lanes which may lead to significant reduction of traffic flow capacity and efficiency, traffic flow disruptions, congestion creation, and increased accident risk. Real-time traffic control by use of green-red traffic signals at the motorway mainstream is proposed in order to achieve safer merging of vehicles entering the work zone and, at the same time, maximize throughput and reduce travel delays. A particular issue addressed in this research is the investigation of the appropriate distance between the merge area and the traffic lights so as to lead, in combination with the employed real-time traffic control strategy, to the most efficient merging of vehicles. The control strategy applied for real-time signal operation is an ALINEA-like PI-type feedback regulator. In order to achieve maximum performance of the control strategy, some calibration of the regulator's parameters may be necessary. The calibration is first conducted manually, via a typical trial-and-error procedure. In an additional investigation, the recently proposed learning/adaptive algorithm AFT is employed in order to automatically fine-tune the regulator parameters. Experiments conducted with a microscopic simulator for a hypothetical work zone infrastructure, demonstrate the potential high benefits of the control scheme.
\end{abstract}

(C) 2012 Published by Elsevier Ltd. Selection and/or peer review under responsibility of the Programme Committee of the Transport Research Arena 2012 Open access under CC BY-NC-ND license.

Keywords: Work zone management; feedback control; merging traffic control; AFT; regulator fine- tuning

\section{Introduction}

Work zones are critical areas of urban and motorway networks and usually require the closure of one or more lanes of the road; as a consequence, the traffic flow needs to merge from a higher number of lanes into a lower number of lanes within a limited space. When the arriving flow reaches or exceeds the

\footnotetext{
${ }^{*}$ Corresponding author. Email address: athina.tymp@gmail.com
} 
reduced downstream capacity, congestion is created, leading to an additional, congestion-induced capacity drop due to the need for vehicles to accelerate from low speeds within the congestion to higher speeds downstream of the congestion head (Papageorgiou et al., 2008). Work zone management aims at safe working conditions for work-zone workers, as well as, safe and efficient passage of vehicles. In the past, several procedures and strategies have been proposed or used to improve traffic conditions at work zones, including speed limitations, as well as signing, markings and particular geometric design, see e.g. (Lin et al., 2004), (FHWA, 2005), (Wei and Pavithran, 2006). More recently, real-time merging traffic control was proposed (Lentzakis et al, 2007), aiming at throughput maximization and minimization of delays in work zones in a similar way as the mainstream traffic flow control concept by (Carlson et al., 2010), albeit by use of traffic lights instead of variable speed limits.

This paper continues on the work of (Lentzakis et al., 2007), improving on the utilized control strategy and investigating the most appropriate positioning of the traffic lights when applying real-time traffic control. The distance between the traffic lights and the merge area is crucial as it affects the vehicles' behavior and particularly the acquired speed when approaching the merge area. It is shown that the appropriate location of the traffic lights may improve the results of merging traffic control, as the capacity drop can be reduced or even eliminated in case of proper merging vehicle speed, and this contributes to a more efficient and safe passage through the merge area.

Another novel issue addressed, is the calibration of the employed regulator parameters for the applied control strategy with particular focus on a recently proposed (Kouvelas, 2011), (Kouvelas et al., 2011) automatic fine-tuning procedure aiming at optimizing the regulator parameters and ensuring best performance of the utilized control strategy.

\section{Work Zone Traffic Control}

\subsection{Merge area}

A typical motorway work zone area is sketched in Fig. 1a. The vehicles arriving on $M$ lanes, must change lanes appropriately within the (typically trapezoidal) merge area (or earlier) so as to fit into the $\mu$ lanes of the exit (where $M$ is higher than $\mu$ ). The merging procedure may be quite complex in terms of the required vehicle maneuvers, especially when the arriving flow is higher than the work zone flow capacity.

The capacity of work zone areas is usually lower than the mainstream motorway capacity due to the drop of one or more lanes at the work zone entrance. Fig. 1b displays a typical flow-density diagram for the merge area, where the flow $q_{\text {out }}$ is the merge area exit flow and $N$ is the number of vehicles included in the merge area. When the number of vehicles $N$ included in the merge area is small, merging conflicts are scarce and swift, while the merge area exit flow $q_{\text {out }}$ is correspondingly low. As the arriving flow, and hence $N$, increase, merging conflicts may increase, but $q_{\text {out }}$ increases as well until, for a specific critical value $N_{\text {cr }}$, the exit flow reaches the downstream capacity $q_{\text {cap }}$. If $N$ increases beyond $N_{\text {cr }}$, merging conflicts become more serious, leading to substantial vehicle decelerations and eventual accelerations that reduce the exit flow to lower values $q_{\mathrm{c}}$, where $q_{\text {cap }}-q_{\mathrm{c}}$ is the capacity drop due to congestion, which is deemed to depend on vehicle acceleration at the congestion head. Under these conditions, real-time control of the arriving flow may be employed in order to maintain the number of vehicles $N$ in the merge area close to its critical value $N_{\text {cr }}$. This is similar to local ramp metering measures (Papageorgiou and Kotsialos, 2002) where, in contrast, only a part of the arriving traffic flow (i.e. only the on-ramp flow) is controlled so as to maximize the merge area throughput. 


\subsection{Control devices and real-time measurements}

Merging traffic control could be applied by use of different control devices aiming at a smooth, safe and efficient merging of vehicles. A possible control device to regulate the arriving flow at work zone areas is traffic lights. A significant issue when applying work zone traffic control, is the proper positioning of the traffic lights so as to achieve the most efficient merging of vehicles. More specifically, the traffic lights should be placed sufficiently upstream from the merge area, so that the vehicles, starting from the traffic lights at low speed, have enough time to reach the appropriate speed for orderly and efficient merging, i.e. a speed that corresponds roughly to capacity flow in Fig. $1 \mathrm{~b}$ (critical speed). Traffic lights can be applied to all lanes simultaneously or to individual lanes separately. If necessary, traffic lights may be installed only on a part of the motorway lanes, while traffic flow on other lanes is allowed to enter the merge area freely (e.g. by-pass lanes for HOV, buses, emergency vehicles).

In order to apply feedback control and maintain the number of vehicles $N$ close to $N_{\text {cr }}$, real-time measurements or estimates of $N$ are needed. A frequently practiced way of estimating $N$ is by use of ordinary loop detectors placed at appropriate positions (Vigos and Papageorgiou, 2008). Alternatively, one may employ occupancy measurements and target a critical occupancy value $o_{\text {cr }}$ (instead of $N_{\text {cr }}$ ) as in ALINEA ramp metering.
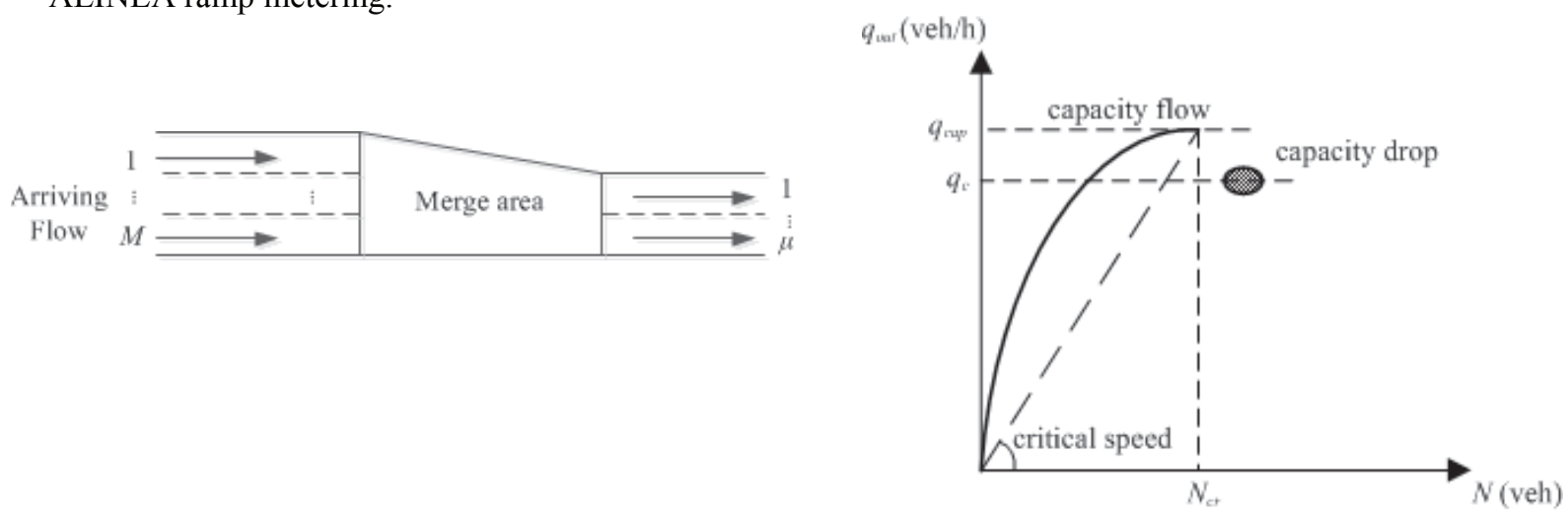

Fig. 1. (a) Typical motorway work zone area and; (b) fundamental diagram of a merge area.

\subsection{Control algorithm}

The control algorithm makes use of real-time measurements or estimates of the number of vehicles $N$ or occupancy $o$ in the network in order to maintain $N \approx N_{\text {cr }}$ or $o \approx o_{\text {cr }}$, which maximizes the merge area exit flow. The feedback algorithm used in this study for merging traffic control is an extension of the local ramp metering strategy ALINEA (Papageorgiou et al., 1991; 1997). ALINEA is an integral (I-type) feedback regulator given by the equation

$$
q(k)=q(k-1)+K_{\mathrm{R}}[\hat{o}-o(k-1)]
$$

where $k=1,2, \ldots$ is the discrete-time index, $q(k)$ denotes the controlled entering flow to be implemented during the next period $k, K_{\mathrm{R}}>0$ is a regulator parameter, $o(k-1)$ is the last measured occupancy (\%) and $\hat{o}$ is a set (desired) value for the downstream occupancy of the motorway. A typical set-value is $\hat{o}=o_{\text {cr }}$ in which case the motorway exit flow becomes close to $q_{\text {cap }}$. The same equation can be used if the number of vehicles $N$ is measured, instead of the occupancy percentage. Within the 
microscopic simulation environment, the performance of the described regulator was sometimes sluggish, and therefore, a proportional-integral (PI-type) regulator is used (Wang and Papageorgiou, 2006), which appears more reliable for the present investigation. The PI-type regulator reads

$$
q(k)=q(k-1)-K_{\mathrm{P}}[N(k)-N(k-1)]+K_{\mathrm{I}}[\hat{N}-N(k)]
$$

where $K_{\mathrm{P}}$ and $K_{\mathrm{I}}$ denote the regulator parameters for the proportional and integral terms, respectively, that must be suitably specified, and $\hat{N}$ is a set (desired) value for the downstream number of vehicles.

The control algorithm is activated at each time interval $T$ (in s) and calculates the entering flow $q(k)$ (in veh/h) to be implemented in the next interval $k$ via appropriate operation of the control devices (traffic lights). There are different possible metering policies to translate the decision of the control strategy (i.e. the flow $q(k))$ into corresponding traffic light settings; e.g. one-car-per-green, $n$-cars-pergreen, full traffic cycle, discrete release rates etc., see (Papageorgiou and Papamichail, 2008). A full traffic cycle policy is employed here so as to maximize the resulting flow capacity of the traffic lights. The flow to be implemented in the next control period $T$ may be distributed equally among the motorway lanes via corresponding individual traffic lights for each lane; while a shift (offset) should be applied for the signal cycle start of each traffic light relative to the cycles of the other traffic lights, so as to enable (to the extent possible) a continuous flow and avoid simultaneous vehicle departures from all lanes (or no departures during red).

\section{Application Setup}

The described real-time work zone merging control concept is implemented, via microscopic simulation, at a hypothetical work zone infrastructure featuring 3 arriving lanes and 2 exiting lanes as depicted in Fig. 2. The total length of the simulated motorway stretch is $5 \mathrm{~km}$ (to accommodate any forming queue length), while the trapezoidal merging area, which is situated $100 \mathrm{~m}$ before the end of the motorway stretch, is $50 \mathrm{~m}$ long. A speed limit of $80 \mathrm{~km} / \mathrm{h}$ is applied along the whole motorway stretch. The capacity $q_{\text {cap }}$ of the motorway upstream of the work zone area is sufficiently high to accommodate the investigated demand scenario, while the downstream capacity is reduced due to the lane drop and was found empirically to amount to $4800 \mathrm{veh} / \mathrm{h}$ (for a traffic flow including $20 \%$ trucks). Another feature of the described infrastructure is that the left-most lane of the motorway, which is the high-speed lane, is reserved only for cars, while trucks are allowed to use only the other two lanes, as in several real motorways (see Fig. 2). For the collection of measurements, for operation or evaluation, detectors have been placed at different positions along the stretch, as displayed in Fig. 2.

The control concept was implemented for a representative demand scenario, which has a duration of 2 hours and trapezoidal profile. At the beginning of the simulation, the average demand at the motorway entrance starts at a low value (50\% of the highest traffic demand). The demand increases gradually within the first 30 minutes, until it reaches a peak demand of $5400 \mathrm{veh} / \mathrm{h}$; and remains at this value for the next 30 minutes. During this time period the traffic demand exceeds the merge area capacity $q_{\text {cap }}$ which is expected to lead to congestion and reduced efficiency of the infrastructure. During the time period $t \in[60 \mathrm{~min}, 90 \mathrm{~min}]$, the demand reduces gradually back to the initial low value $(50 \%$ of the highest demand) and remains at that value for the rest $30 \mathrm{~min}$, i.e. until the end of the simulation. Any queues must be resolved at the end for all scenarios, to obtain comparable results.

The vehicle types included in the demand scenario are cars and trucks. The trucks represent an average of $20 \%$ of the total traffic demand, and this percentage remains constant (in average) throughout the simulation. While determining $N$ for the control algorithm, trucks are counted as equivalent to two cars. 
The regulator (equation (2)) is activated every $T=30 \mathrm{~s}$ and receives the real-time measurement of the number of vehicles $N$ included in the merge area (calculated from detectors 1 and 3 in Fig. 2) to calculate the entering flow $q(k)$ to be implemented in the next control period $k$ so as to maintain $N \approx N_{\text {cr }}$. The new entering flow to be implemented is not allowed to exceed the range $q \in[4000,6000] v e h / h$, i.e. a minimum and maximum flow, respectively. Specifically, the minimum admissible flow $q_{\min }$ was selected lower than the downstream capacity to enable a sufficient margin for regulator action; for the same reason the maximum admissible flow $q_{\max }$ was selected sufficiently large and higher than $q_{\text {cap }}$. The traffic cycle is fixed and equal to the control interval (30 s); while the green and red phases are calculated appropriately to implement the ordered flow $q(k)$, with a minimum red phase of $3 \mathrm{~s}$ being considered for safety reasons. More specifically, the flow $q(\mathrm{veh} / \mathrm{h})$ is translated into a corresponding green phase $G$ (in s) via the following equation

$$
G=(q \cdot T) / S
$$

where $S$ (in veh/h) is the saturation flow, with subsequent application of constraints for the green phase $G$ mentioned earlier. In particular, the minimum-red constraint of $3 \mathrm{~s}$ leads to a maximum green $G_{\max }=27 \mathrm{~s}$, while, for the given $q_{\min }$ and a saturation flow of $2000 \mathrm{veh} / \mathrm{h}$, the minimum green resulting from (3), is $6 \mathrm{~s}$. The same green phase is implemented at all motorway lanes, albeit with an offset of the cycle start as mentioned earlier. In view of the minimum-red constraint, the maximum implementable flow resulting from (3) is $5400 \mathrm{veh} / \mathrm{h}$.

The specification of appropriate regulator parameter values was conducted manually, via trial-anderror. Specifically, various sets of values were tested through a series of simulation runs considering a specific position for the traffic lights. Given that the employed regulator is not very sensitive to the distance between the measurement point and the control device thanks to its feedback structure, the parameter values resulted from this investigation should work equally well for different positions of the traffic lights.

In order to specify a preliminary but reasonable position for the traffic lights, a primary investigation was conducted, using a fixed flow rate for the traffic lights and setting the traffic lights at different locations upstream of the work zone area, from $50 \mathrm{~m}$ up to $300 \mathrm{~m}$ in steps of $50 \mathrm{~m}$. The distance for which vehicles had enough time to acquire a speed close to the critical value, before reaching the merge area, was around $200 \mathrm{~m}$. Therefore, the regulator fine-tuning experiments were conducted by positioning the traffic lights $200 \mathrm{~m}$ upstream from the merge area (Fig. 2).

The described infrastructure was simulated by use of the microscopic simulator AIMSUN v.6.0.6 (TSS, 2009), using the simulator's default parameters and a simulation time step of $0.1 \mathrm{~s}$. The implementation of the control strategy was done via the AIMSUN API (Application Programming Interface), that allows the user to emulate a real-time control environment. Specifically, the simulator delivers at every control period $T$ the number of vehicles $N$ between detectors 1 and 3; based on these measurements, the control software calculates the corresponding traffic light settings and returns them to the micro-simulator for application. Since the AIMSUN simulator model is stochastic, different replications with different random seeds may produce different results. For this reason, 10 replications with different random seeds were carried out for each examined scenario. 


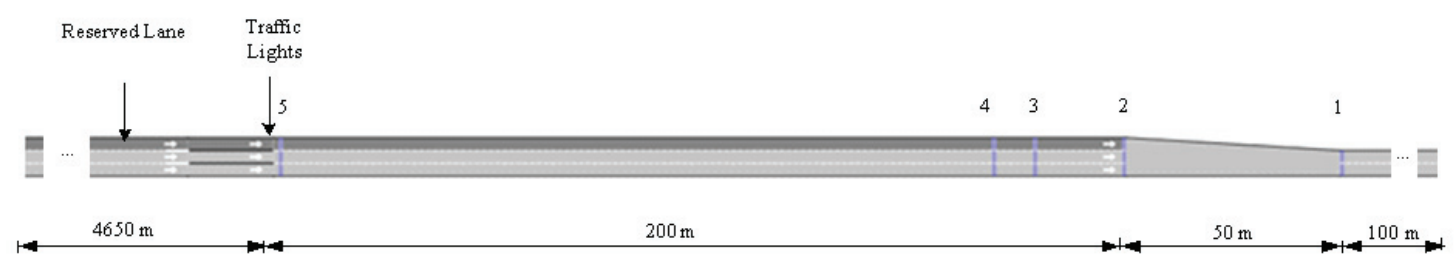

Fig. 2. The work zone infrastructure under consideration. (Numbers correspond to emulated detector locations.)

\section{Simulation Results}

\subsection{No-control case}

In the no-control case, arriving vehicles enter the merge area and exit without any serious problem, as long as the arriving demand is sufficiently low. When the demand increases (peak period) beyond the work zone capacity, vehicle merging conflicts are observed that lead to vehicle decelerations and formation of congestion. Congestion spills back several kilometers, but without ever reaching the simulated network entrance. The mean of the resulting average vehicle delays (AVD) (in $\mathrm{s} / \mathrm{veh} / \mathrm{km}$ ) for 10 replications is 38.1 while the minimum and maximum values are 24.7 and 51.7, respectively.

The trajectories in Figs. 3a and 3b display the merge area outflow $q_{\text {out }}$ (collected from detector 1) and the number of vehicles in the merge area, respectively, for one particular simulation run with $\mathrm{AVD}=37.97 \mathrm{~s} / \mathrm{veh} / \mathrm{km}$, which is closest to the mean AVD of the 10 replications. Until about $t=40 \mathrm{~min}$, the number of vehicles $N$ in the merge area is slowly increasing (as a consequence of the increasing demand), while the merge area outflow is seen to follow the increase of arriving demand, reaching approximately $5000 \mathrm{veh} / \mathrm{h}$ in average. At $t=40 \mathrm{~min}$, the number of vehicles in the merge area increases steeply due to serious merging conflicts that lead to a speed breakdown, and this congested traffic situation becomes stationary until $t=110 \mathrm{~min}$. The outflow during this time period is reduced to around $4150 \mathrm{veh} / \mathrm{h}$ in average due to the merge area congestion (capacity drop). Fig. 4a shows a snapshot of the simulated stretch at $t=60 \mathrm{~min}$ with the formed congestion. After $t=110 \mathrm{~min}$, when the queue dissolves, the number of vehicles in the merge area is seen to drop, and the outflow reduces to lower values due to the decreased demand. Vehicle speed measurements collected upstream of the merge area, at detector 4, indicate that, during the maximum traffic demand (peak period), there is a serious speed drop down to around $20 \mathrm{~km} / \mathrm{h}$ in average, while in the rest of the simulation horizon the average vehicle speed is around $82 \mathrm{~km} / \mathrm{h}$ in average.

\subsection{Merging control case}

When real-time merging traffic control is applied, the maximum admissible flow $q_{\max }=6000 \mathrm{veh} / \mathrm{h}$ is ordered by the regulator for as long as the number of vehicles $N$ in the merge area is lower than the set value $\hat{N}$ in PI-ALINEA's equation (2). As the demand increases, $N$ increases as well, and when $N(k)$ approaches $\hat{N}$, the controller starts its actual operation aiming at maintaining $N(k)$ close to $\hat{N}$. At this time, a queue is formed upstream of the traffic lights (since the arriving demand is higher than the work zone capacity) which propagates backwards, but without reaching the entrance of the simulated system. Fig. $4 \mathrm{~b}$ shows a snapshot of the simulated stretch at $t=60 \mathrm{~min}$ with the formed queue upstream of the traffic lights. 

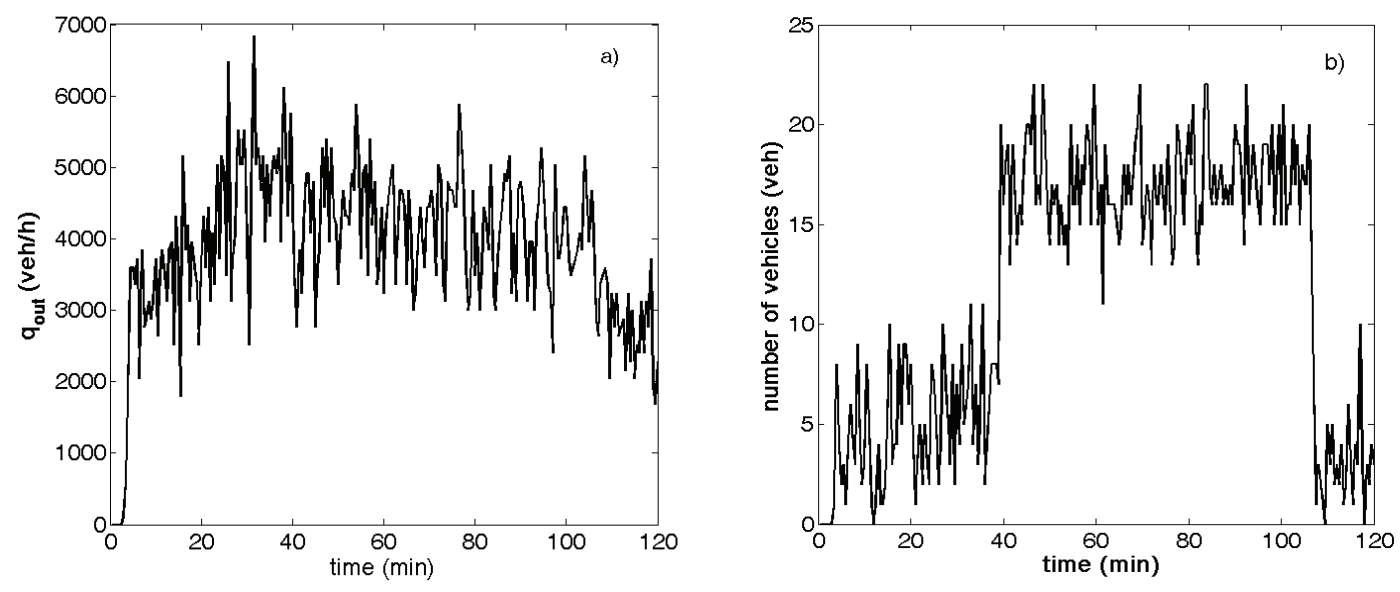

Fig. 3. (a) Outflow $q_{\text {out }}$ and; (b) number of vehicles $N$ in the no-control case.

A critical question at this point is the proper choice of the regulator parameters $K_{\mathrm{P}}, K_{\mathrm{I}}$ and of the setpoint $\hat{N}$. The regulator parameters may be manually fine-tuned following some practical trial-and error rules from Control Engineering; once appropriate values have been found, the regulator is known to be little sensitive to related moderate variations. The manually derived values of regulator parameters $K_{\mathrm{P}}$ and $K_{\mathrm{I}}$ are $150 \mathrm{~h}^{-1}$ and $6 \mathrm{~h}^{-1}$, respectively. The value of $\hat{N}$, on the other hand, should be selected such that the work zone throughput is maximized, according to Fig. 1b. In a field investigation, this may be achieved by gradually incrementing $\hat{N}$ and monitoring the measured outflow, until a maximum throughput is obtained. In the current case, the investigation of the $\hat{N}$ value is carried out through a series of simulation experiments with different (integer) $\hat{N}$ values within the range $\hat{N} \in[6,20]$ veh. For each investigated $\hat{N}$ value, the mean AVD of 10 replications is obtained. Fig. 5a displays, for every investigated $\hat{N}$ value, the corresponding AVD values for the 10 replications as well as the mean, minimum and maximum AVD of all replications. The mean, minimum and maximum AVD values for 10 replications of the no-control case are also displayed on the same figure for comparison. According to the displayed results, the mean AVD value is minimized in the range of $\hat{N} \in[9,14]$ veh, and, particularly for $\hat{N}$ equal to $11 \mathrm{veh}$, it takes the lowest value, which corresponds to the critical value mentioned earlier. For lower $\hat{N}$ values, the system operates at undercritical conditions (Fig. 1b) and the merge area "starves for flow"; while, for higher set points, the merging conflicts are increasingly frequent and serious, leading to reduced throughput. It is also noteworthy that the mean AVD for all investigated $\hat{N}$ is significantly lower than the corresponding value of the no-control scenario. Particularly, for $\hat{N}=11$ veh, the mean AVD is $14.25 \mathrm{~s} / \mathrm{veh} / \mathrm{h}$, which is $63 \%$ lower than the mean AVD of the no-control case.

Figs. $5 \mathrm{~b}$ and $5 \mathrm{c}$ display the merge area outflow $q_{\text {out }}$ and the number of vehicles in the merge area, respectively, for one particular replication with $\hat{N}=11 \mathrm{veh}$ and $\mathrm{AVD}=13.83 \mathrm{~s} / \mathrm{veh} / \mathrm{km}$, which is closest to the mean AVD value of the corresponding 10 replications. The number of vehicles in the merge area is maintained around the set-point $\hat{N}=11 \mathrm{veh}$ (red dashed line in the figure) during the peak period. The observed spikes are due to stochastic arrivals, but also due to some occasional vehicle merging conflicts that may occur and lead to vehicle decelerations in the merge area; the appropriate reaction of the regulator in such cases, guarantees that the number of vehicles in the merge area remains around the setpoint in average. The outflow $q_{\text {out }}$ maintains its average value around $4850 \mathrm{veh} / \mathrm{h}$ during the peak period 
Fig. 4. Screenshot of simulation ( $t=60$ ): (a) without control ; (b) with merging traffic control.

(between $t=30 \mathrm{~min}$ and $t=60 \mathrm{~min}$ ), and beyond the peak period for some 20 more minutes because of the queued vehicles at the traffic lights. This marks a reduction of the congested period by $30 \mathrm{~min}$ (or around $43 \%$ ) compared to the no-control case. The mean vehicle speed upstream of the merge area is significantly increased during the peak hours to $70 \mathrm{~km} / \mathrm{h}$, except for the occasional departures to lower values due to corresponding merging conflicts.

\subsection{Investigation of the position of the traffic lights}

The location of the traffic lights should be sufficiently upstream of the merge area in order to allow for the vehicles to accelerate and pass through the merge area efficiently, i.e. without major decelerations that give rise to the capacity drop observed in the no-control case. Considering a range of possible distances from 30 up to $400 \mathrm{~m}, 10$ replications were simulated for each of them. The obtained mean AVD values as well as the acquired vehicle speed when approaching the merge area (save the occasional drops due to short-lasting merging conflicts) are the main evaluation criteria for the determination of the optimum position of the traffic lights. Fig. 6a depicts, for every investigated traffic lights position, the resulting AVD values for the 10 replications, as well as the mean, minimum and maximum AVD of all replications. The mean, minimum and maximum AVD values of the 10 replications of the no-control case are also displayed on the same figure for comparison. The mean average vehicle delay is low and virtually constant when the traffic lights are located $150 \mathrm{~m}$ upstream of the merge area or more. In contrast, when traffic lights are placed closer to the merge area, higher AVD values are seen to result. Fig. $6 \mathrm{~b}$ displays the trajectories of the speed measurements collected upstream of the merge area, only for
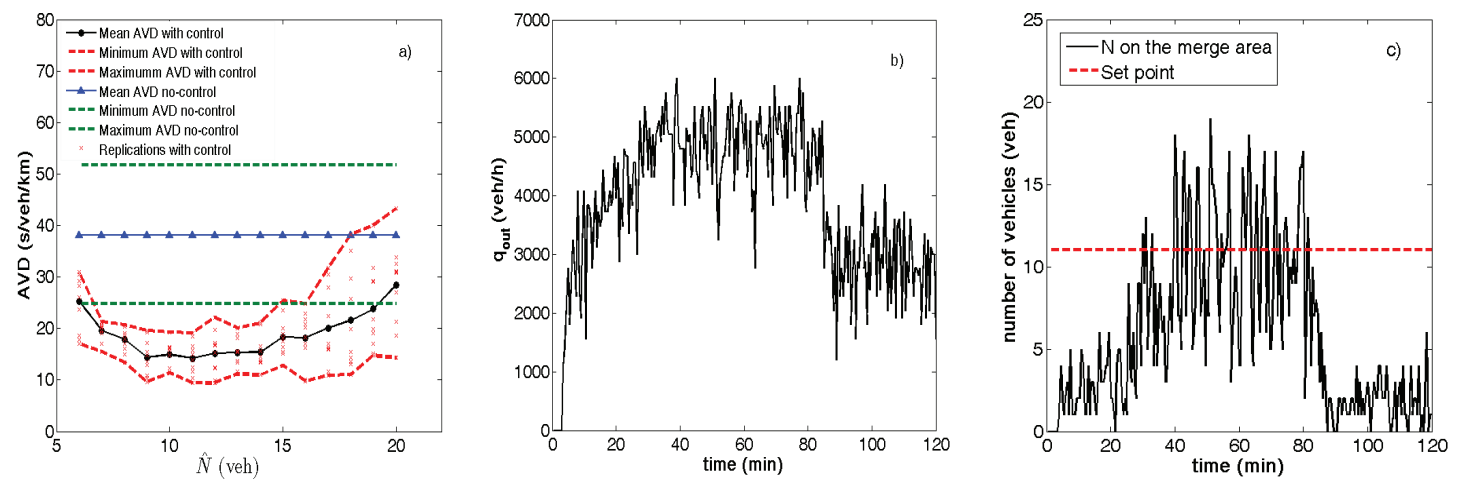

Fig. 5. (a) Average vehicle delay versus $\hat{N}$ values with and without control; (b) outflow $q_{\text {out }}$ and; (c) number of vehicles $N$ for the control case. 
selected traffic lights positions, for readability. When the traffic lights are placed very close to the merge area, e.g. at $30 \mathrm{~m}$ or $50 \mathrm{~m}$, vehicles do not have sufficient time to accelerate, and indeed it can be observed that the mean speed value during the peak period is quite low, i.e. lower than $45 \mathrm{~km} / \mathrm{h}$. For a distance equal to $100 \mathrm{~m}$, the merging vehicle speed increases, reaching $60 \mathrm{~km} / \mathrm{h}$ in average. For the traffic lights position of $200 \mathrm{~m}$ upstream of the merge area, the achieved merging vehicle speed has increased to a mean value around $70 \mathrm{~km} / \mathrm{h}$. For even longer distances, e.g. $400 \mathrm{~m}$, the speed is even more increased to around $80 \mathrm{~km} / \mathrm{h}$. The observed occasional speed drops are due to temporal sharp vehicle conflicts. Apparently, distances less than $150 \mathrm{~m}$ are less appropriate, and, particularly for distances less than $50 \mathrm{~m}$, the system performance comes quite close to the no-control case, because the capacity drop is only partially avoided. For distances more than $200 \mathrm{~m}$, there is no further improvement, since the critical merging speed has been reached. Thus, $200 \mathrm{~m}$ is the most appropriate distance, as it is preferable to have the traffic lights closer to the merge area.
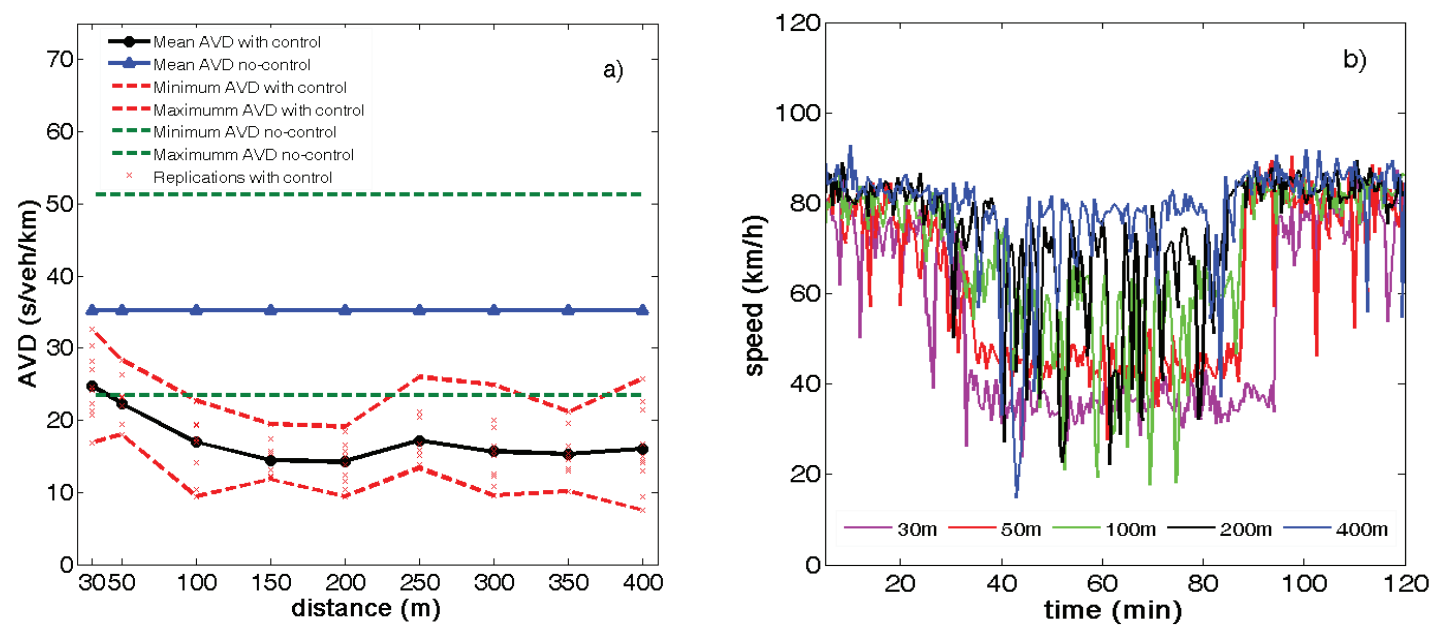

Fig. 6. (a) Average vehicle delay; and (b) Speed versus time for different traffic lights positions.

\section{Application of AFT to the merging control strategy}

Recently, a learning/adaptive algorithm called AFT (Adaptive Fine-Tuning) was proposed (Kouvelas, 2011; Kouvelas et al., 2011) to enable automatic fine-tuning of traffic control systems (TCS), so as to reach the best measurable performance that is achievable with the applied control strategy. In this section, the automatic fine-tuning method is employed for the motorway work-zone merging control concept in order to automatically fine-tune the regulator parameters of the PI-ALINEA control strategy. The original algorithm employs a polynomial-like approximator (similar to a neural network) that approximates, based exclusively on available measurements, the unknown nonlinear performance function of the problem. For this application, a polynomial approximator as well as a Support Vector Machine (SVM) model (see Burges, 1998) are used. An SVM model was recently used by Giannakis, Kontes, Kosmatopoulos \& Rovas (2011) within the AFT frame in order to fine-tune the parameters of a building's controller. The performance function (to be minimized) is the average vehicle delay (AVD). The AFT algorithm (Fig. 7) runs iteratively, where one iteration corresponds to the duration of the demand scenario (2 hours), or of one day in field applications. At each iteration, the collected measurements are used to update and enhance the performance function approximation; and eventually to modify the regulator parameters so as 
to decrease the value of the approximated performance function; until convergence to an optimal solution (local minimum) is achieved.

The AFT algorithm is started with some initial values for the parameters to be tuned. When using an SVM approximator, the algorithm convergences fast to a close local minimum of the performance function and remains there; while the original AFT may feature more significant "jumps" in the parameter space, with correspondingly stronger fluctuations of the performance function. In order to use SVM, a sufficient initial set of training data is needed to be available for fitting. Therefore, the original AFT (using the polynomial approximator) is applied for the first 10 iterations, before switching to the SVM usage. In particular, for the first 10 iterations, AFT explores a wide region of alternative sets of parameters; following which the SVM is used and the algorithm converges to a close local minimum without exploring other feasible regions.

In a first experiment, the AFT algorithm is applied for a control scenario with the initial values of the tunable parameter set as following: $K_{\mathrm{P}}=150 \mathrm{~h}^{-1}, K_{\mathrm{I}}=6 \mathrm{~h}^{-1}$ and $\hat{N}=11 \mathrm{veh}$. These are the optimized parameter values derived via the trial-and-error procedure, which means that these values represent already a "good" starting point for the AFT algorithm. Fig. 8a displays the AVD values trajectory delivered for one run of the AFT algorithm. Some strong fluctuations are observed in the first 20 iterations, but during the rest of the fine-tuning period the AFT algorithm achieves keeping the AVD at low values. The AFT algorithm converges to the following values for the parameters: $K_{\mathrm{P}}=80.88 \mathrm{~h}^{-1}$, $K_{\mathrm{I}}=2.95 \mathrm{~h}^{-1}$ and $\hat{N}=8.37 \mathrm{veh}$. The delivered set of values was then applied within the work-zone control concept in order to compare the system performance before and after the use of the AFT algorithm. The resulting mean AVD for 10 replications is $13.0 \mathrm{~s} / \mathrm{veh} / \mathrm{km}$, which is around $9 \%$ lower than the mean AVD of the initial control scenario before using the AFT algorithm.

In a second AFT experiment, the starting values for the tunable parameters were set $K_{\mathrm{P}}=50 \mathrm{~h}^{-1}$, $K_{\mathrm{I}}=10 \mathrm{~h}^{-1}$ and $\hat{N}=10 \mathrm{veh}$. This set of parameters was arbitrarily selected in order to investigate the algorithm's behavior. The simulated 10-replication results for the control scenario using these parameter values lead to quite high AVD values, with a mean AVD around $21 \mathrm{~s} / \mathrm{veh} / \mathrm{km}$. Fig. $8 \mathrm{~b}$ presents the trajectory of the AVD values during the AFT fine-tuning process. It is visible that the obtained AVD values perturbate due to the search process of the AFT algorithm, but finally AFT achieves to locate a satisfactory set of parameters that leads to low AVD values. The AFT algorithm converges to $K_{\mathrm{P}}=73.83$ $\mathrm{h}^{-1}, K_{\mathrm{I}}=4.53 \mathrm{~h}^{-1}$ and $\hat{N}=8.59 \mathrm{veh}$, and the corresponding mean AVD for 10 replications is 14.09 $\mathrm{s} / \mathrm{veh} / \mathrm{km}$, which is around $34 \%$ lower than the mean AVD of the starting control scenario before using AFT. The performance with the obtained parameter values is even slightly better than the manually finetuned regulator parameters.

In a third AFT experiment, the starting values for the tunable parameters were $K_{\mathrm{P}}=200 \mathrm{~h}^{-1}$, $K_{\mathrm{I}}=50 \mathrm{~h}^{-1}$ and $\hat{N}=50$ veh with a corresponding mean AVD, for 10 replications, around $36 \mathrm{sec} / \mathrm{veh} / \mathrm{km}$. These values correspond to a very "bad" set of regulator parameters and they are selected in order to investigate the performance of the AFT algorithm and the values that it is going to converge to, under very unfavorable starting conditions. Fig. 8c presents the trajectory of the AVD values during the finetuning process. During the first iterations, the AVD values are quite high; however, after the $20^{\text {th }}$ iteration, the AFT algorithm manages to lead and keep the AVD at lower values. The AFT algorithm converges to $K_{\mathrm{P}}=204.18 \mathrm{~h}^{-1}, K_{\mathrm{I}}=31.32 \mathrm{~h}^{-1}$ and $\hat{N}=12.13 \mathrm{veh}$. The obtained values of $K_{\mathrm{P}}$ and $K_{\mathrm{I}}$ are relatively close to the initial ones, but the value $\hat{N}$ has been reduced a lot. The resulting mean AVD for 10 replications is $15 \mathrm{~s} / \mathrm{veh} / \mathrm{km}$ which is $57 \%$ lower than the mean AVD of the starting control scenario, albeit slightly worse than in the manually fine-tuned case.

The described experiments show that the AFT algorithm can improve the system performance independently of the starting points. Nevertheless, the selection of, at least roughly, appropriate starting 
values for the regulator parameters (e.g. derived from manual fine-tuning) may be necessary in order to achieve best performance of the utilized control strategy.

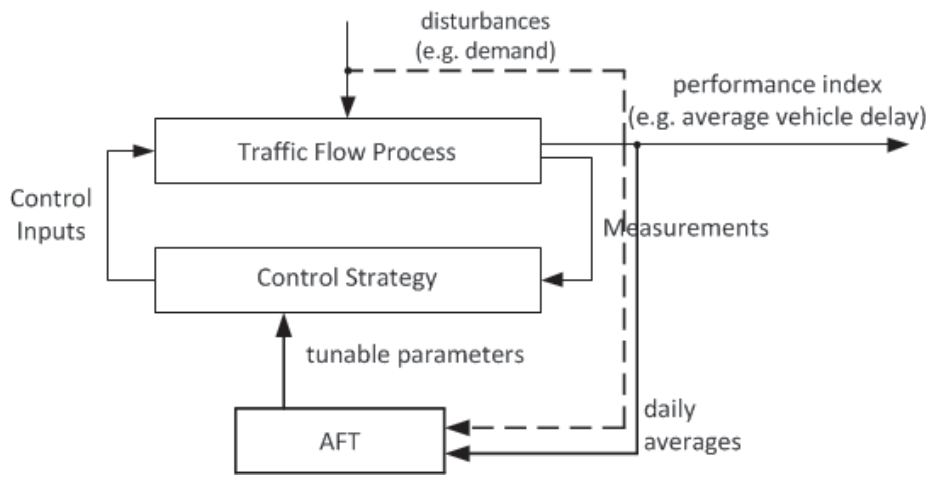

Fig. 7. Working principle of AFT for automatic calibration.
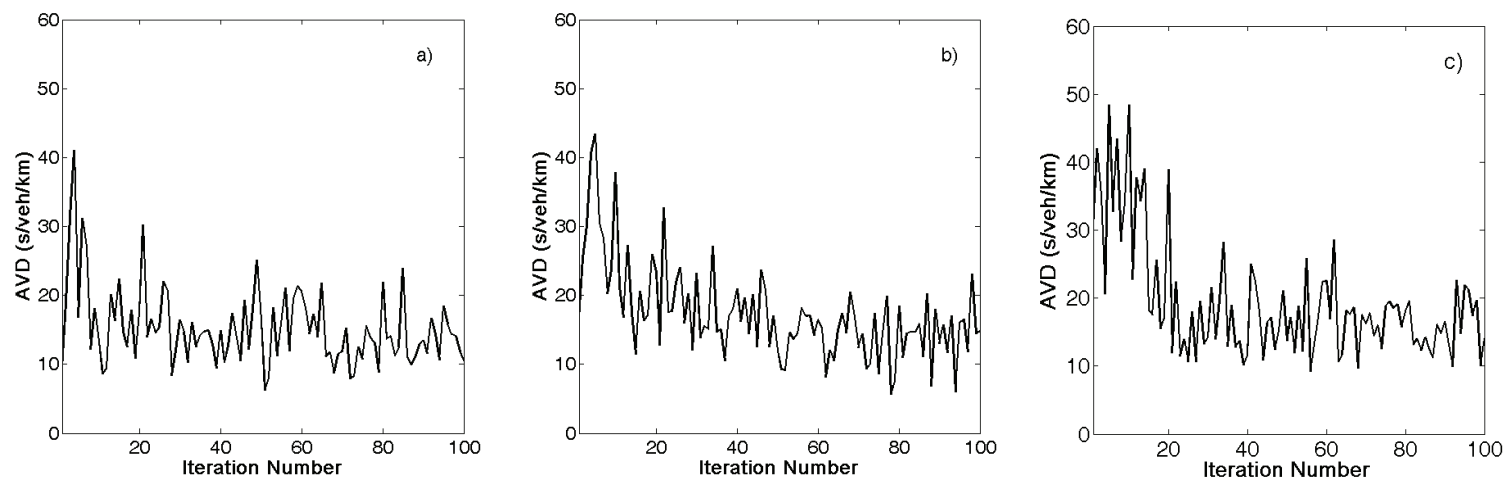

Fig. 8. (a) Average vehicle delay for control scenario 1; (b) Average vehicle delay for control scenario 2; (c) Average vehicle delay for control scenario 3 .

\section{Conclusions}

A control scheme was developed for real-time merging traffic control at work zones with lane drop, and was applied to a hypothetical work zone motorway infrastructure within a microscopic simulation environment. The control algorithm used for work zone management is an extension of the well-known local ramp metering strategy ALINEA, while the control devices to implement the control algorithm decisions are traffic lights located sufficiently upstream of the work zone area. The reported research addressed the appropriate distance between the traffic lights and the merge area, and demonstrated its significance for throughput maximization (or equivalently delay minimization) via avoidance of the capacity drop. Although safety issues were not explicitly addressed, we believe that the presented scheme should also improve on safety due to smoother merging. A further investigation in this research was related to the fine-tuning procedure needed for the calibration of the control algorithm parameters. In particular, after the manually conducted fine-tuning procedure, the recently proposed learning/adaptive algorithm AFT was applied in order to seek for better regulator parameter values which lead to improved performance of the utilized control strategy. The significance of this investigation is that the AFT 
algorithm can be applied similarly in the field, based on real measurements, to optimize the control system performance.

\section{References}

Burges, C. J. C., (1998). A Tutorial on support vector machines for pattern recognition. Data Mining and Knowledge Discovery 2, pp. 121-167.

Carlson, R. C., Papamichail, I., Papageorgiou, M., and Messmer, A., (2010). Optimal mainstream traffic flow control involving variable speed limits and ramp metering. Transportation Science, 18, pp.193-210.

FHWA, (2005). Developing and Implementing Transportation Management Plans for Work Zones. Report of the Federal Highway Administration, U.S.. Department of Transportation.

Giannakis, G. I., Kontes, G. D., Kosmatopoulos, E. B., \& Rovas, D. V., (2011). A model-assisted adaptive controller fine-tuning methodology for efficient energy use in buildings. 19th IEEE Mediterranean Conference on Control and Automation, Corfu, Greece, June, pp. 49-54.

Kouvelas, A., (2011). Adaptive Fine-Tuning for Large-Scale Nonlinear Traffic Control Systems (PhD thesis). Technical University of Crete, Chania, Greece.

Kouvelas, A., Aboudolas, K., Kosmatopoulos, E. B., and Papagorgiou, M., (2011). Adaptive performance optimization for largescale traffic control systems. IEEE Trans. on Intelligent Transportation Systems, 12 (4), pp. 1434-1445.

Lentzakis, A. F., Spiliopoulou, A. D., Papamichail, I.,and Papageorgiou, M., (2008). Real-time work zone management for throughput maximization. 87th Transportation Research Board Annual Meeting, Washington, DC, January 13-17, paper 080772 .

Lin, P. W., Kang, K. P., and Chang, G. L., (2004). Exploring the effectiveness of variable speed limit controls on highway workzone operations. Journal of Intelligent Transportation Systems, 8, pp. 155-168.

Papageorgiou, M., Haj-Salem, H., and Blosseville, J-M.,(1991). ALINEA: A local feedback control law for on-ramp metering. Transportation Research Record 1320, pp. 58-64.

Papageorgiou, M., Haj-Salem, H., and Middelham, F., (1997). ALINEA local ramp metering: summary of field results. Transportation Research Record 1603, pp. 90-98.

Papageorgiou, M., and Papamichail, I.,(2008). Overview of traffic signal operation policies for ramp metering. Transportation Research Record 2047, pp. 28-36.

Papageorgiou, M., Papamichail, I., Spiliopoulou, A. D., and Lentzakis, A. F., (2008). Real-time merging traffic control with applications to toll plaza and work zone management. Transportation Research Part C, 16, pp. 535-553.

Papageorgiou, M., and Kotsialos, A., (2002). Freeway ramp metering: an overview. IEEE Trans. on Intelligent Transportation Systems 3, pp. 271-281.

TSS-tranport simulation systems, (2009). Aimsun Users Manual Version 6.

Vigos, G., Papageorgiou, M., and Wang., Y., (2008). Real-time estimation of vehicle-count within signalized links. Transportation Research Part C, 16, pp. 18-35.

Wang, Y. , and Papageorgiou, M., (2006). Local ramp metering in the case of distant downstream bottlenecks. IEEE Intelligent Transportation Systems Conference, Toronto, Canada, September 17-20.

Wei, H., and Pavithran, M., (2006). Concept of dynamic merge metering approach for work zone traffic control. 11th IFAC Symposium on Control in Transportation Systems. Delft, The Netherlands, August 29-31, pp. 374-379. 